\title{
Search for nuclearites with the KM3NeT detector
}

\section{Alice Păun ${ }^{a, b, *}$, Gabriela Păvălaș ${ }^{a}$ and Vlad Popa ${ }^{a}$ on behalf of the KM3NeT \\ Collaboration}

(a complete list of authors can be found at the end of the proceedings)

${ }^{a}$ Institute of Space Science,

Atomistilor 409, Magurele, Romania

${ }^{b}$ Faculty of Physics, University of Bucharest, Atomistilor 405, Magurele, Romania

E-mail: alice.paun@spacescience.ro

Strange quark matter (SQM) is a hypothetical type of matter composed of almost equal quantities of up, down and strange quarks. In [1], Edward Witten presented the SQM as a denser and more stable matter that could represent the ground state of Quantum Chromodynamics (QCD). Massive SQM particles are called nuclearites. These particles could have been produced in violent astrophysical processes, such as neutron star collisions and could be present in the cosmic radiation. Nuclearites with masses greater than $10^{13} \mathrm{GeV}$ and velocities of about $250 \mathrm{~km} / \mathrm{s}$ (typical galactic velocities) could reach the Earth and interact with atoms and molecules of sea water within the sensitive volume of the deep-sea neutrino telescopes. The SQM particles can be detected with the KM3NeT telescope (whose first lines are already installed and taking data in the Mediterranean Sea) through the visible blackbody radiation generated along their path inside or near the instrumented area. In this work the results of a study using Monte Carlo simulations of down-going nuclearites are discussed.

The $37^{\text {th }}$ International Cosmic Ray Conference

12-23 July, 2021

Berlin, Germany

*Speaker

(C) Copyright owned by the author(s) under the terms of the Creative Commons

Attribution-NonCommercial-NoDerivatives 4.0 International License (CC BY-NC-ND 4.0). 


\section{Introduction}

Strange Quark Matter (SQM) was proposed in 1980 by Edward Witten [1] as a hypothetical type of matter consisting of nearly equal quantities of up, down and strange quarks. This type of matter has a density slightly higher than that of nuclear matter and is completely stable. In the same paper, Witten showed that SQM matter could represent the ground state of Quantum Chromodynamics (QCD), by demonstrating that three flavor quark matter have a smaller energy than two flavor quark matter, because of the extra Fermi well available for the strange quark. Light lumps of SQM $\left(\mathrm{A}>10^{7}\right)$ are usually called strangelets, and the heavier ones are called nuclearites. Nuclearites could have been produced in violent astrophysical phenomena, like neutron star collisions and could be present in the cosmic radiation. Massive SQM particles with masses greater than $10^{13} \mathrm{GeV}$ and velocities of about $250 \mathrm{~km} / \mathrm{s}$ at the entry in Earth's atmosphere could have enough energy to reach the Earth and interact with the atoms of sea water within the sensitive volume of a neutrino telescope placed on the bottom of the Mediterranean Sea. In the following will be presented the characteristics of nuclearites, the detection principle and the results of a preliminary study using Monte Carlo simulations of nuclearites.

\section{Nuclearites}

The density of the SQM matter is estimated at approximately $3.6 \times 10^{14} \mathrm{~g} / \mathrm{cm}^{3}$ in [2], which is slightly higher than the density of nuclear matter and neutron stars [3, 4]. Composed of up, down and strange quarks, nuclearites have a positive residual charge which is compensated by electrons, thus resulting a neutral massive particle. The structure of nuclearites with radii $<1 \AA$ (Angstrom) is very similar to the structure of nuclear atoms (Fig. 1). They would have a SQM nucleus surrounded by an electronic shell, that spreads beyond the nucleus shell by at least an electron Compton wavelength [3]. Larger nuclearites would be more similar to a neutron star, and their electron component would be distributed within the SQM nucleus towards its surface.
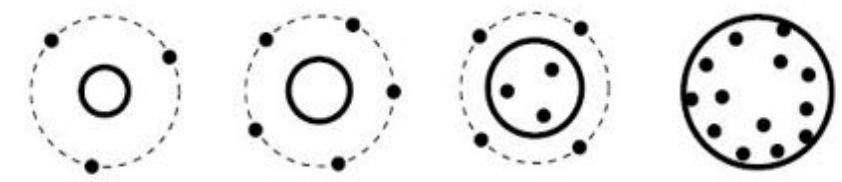

$\mathrm{R}=10^{2} \mathrm{fm}$

$$
\mathrm{R}=10^{3} \mathrm{fm}
$$
$\mathrm{M}=10^{9} \mathrm{GeV}$

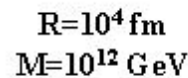

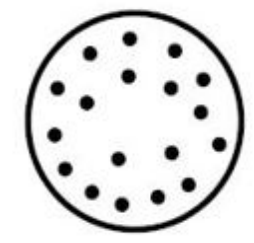

$\mathrm{R}=10^{6} \mathrm{fm}$ $\mathrm{M}=10^{18} \mathrm{GeV}$

Fig. 1: Dimensions and masses (in $\mathrm{GeV}$ ) of the nuclearites and their internal structure. The radii are in logarithmic scale. The electrons are illustrated by black dots. [4]

Massive SQM particles with galactic velocities do not interact with atoms through direct nuclear interactions, because of the Coulomb repulsion. The relevant interaction mechanism of nuclearites with matter is through elastic and quasi-elastic collisions. In transparent media (such as water), a fraction of the energy emitted through the elastic and quasi-elastic collisions of these particles with the atoms encountered is dissipated as blackbody radiation in the visible spectrum. 
This fraction of energy, the luminous efficiency, is estimated in the case of water to $\eta \approx 3 \cdot 10^{-5}$. This allows the search of nuclearites using neutrino telescopes.

The energy loss of massive SQM particles through elastic collisions can be described using the following expression [3]:

$$
\frac{d E}{d x}=-\sigma \rho v^{2}
$$

where $\rho$ is the medium density, $\mathrm{v}$ is the nuclearite velocity and $\sigma$ is the cross-section of the nuclearite, described as follows [3]:

$$
\sigma=\left\{\begin{array}{l}
\pi\left(\frac{3 M_{N}}{4 \pi \rho_{N}}\right)^{2 / 3} \mathrm{~cm}^{2}, \text { for } \mathrm{M}_{N} \geq 8.4 \times 10^{14} \mathrm{GeV} \\
\pi \cdot 10^{-16} \mathrm{~cm}^{2}, \text { for } \mathrm{M}_{N}<8.4 \times 10^{14} \mathrm{GeV}
\end{array}\right.
$$

where $\mathrm{M}_{\mathrm{N}}$ and $\rho_{\mathrm{N}}$ are the mass and density of the nuclearite, respectively.

The velocity of the particle decreases exponentially with the traveled distance L. The propagation of nuclearites through atmosphere and water is described by the expression [3]:

$$
v(L)=v_{0} \cdot e^{\frac{-\sigma}{M_{N}} \cdot \int_{0}^{L} \rho d x}
$$

where $\mathrm{v}(\mathrm{L}), \mathrm{v}_{0}$ are the final and initial velocity, respectively, $\mathrm{M}_{\mathrm{N}}$ is the mass of the nuclearite and $\rho$ is the density of the traversed medium.

Since the nuclearites lose energy by elastic collisions with the atoms of the medium, and through these collisions massive SQM particles overheats the medium and generate blackbody radiation in the visible spectrum, the light emitted in these interactions can be detected by the PMTs of an underwater neutrino telescope. This light is then converted in electric charge, the pulse is digitized and sent to the shore station using optical fibers. At the shore, the signal is analyzed and filtered.

The number of photons emitted in the nuclearite interactions per unit length is described by the following relation [3]:

$$
\frac{d N_{\gamma}}{d x}=\eta_{\text {water }} \cdot \frac{d E / d x}{\left\langle E_{\gamma}\right\rangle}
$$

where $\eta_{\text {water }}=3 \cdot 10^{-5}$ is the luminous efficiency and $E_{\gamma}=3.14 \mathrm{eV}$ is the average energy of the photons emitted.

Considering the previous expression (4), in Fig. 2 is shown the result of a study for the detection of massive downgoing SQM particles made for ORCA configuration using only one Detection Unit with one Digital Optical Module at each level (upper, middle and lower). Also, a downgoing event with a vertical trajectory at the distance of $1 \mathrm{~m}$ from the DU was considered. The number of emitted visible photons per unit of length is represented as a function of the nuclearite mass (in $\mathrm{GeV}$ ). 


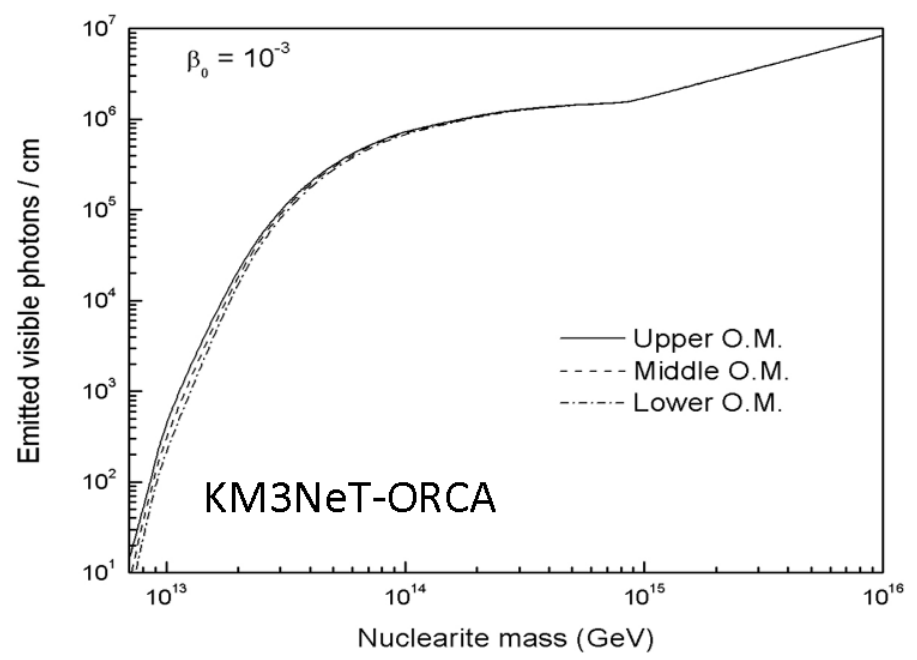

Fig. 2: The number of emitted visible photons per unit of length as a function of the nuclearite mass.

\section{KM3NeT detector}

The detector consists of two large volume photomultiplier (PMT) arrays, ARCA (Astroparticle Research with Cosmics in the Abyss) and ORCA (Oscillation Research with Cosmics in the Abyss), placed at the bottom of the Mediterranean Sea in Italy (3500 m) and France (2475 m), respectively (Fig. 3). Either configuration will be composed by 115 Detection Units (DUs), each DU containing 18 Digital Optical Modules (DOMs). A DOM consists of 31 PMTs distributed on the internal surface of a glass sphere, and several sensors. All the DUs are connected to Junction Boxes (JB) placed on the seabed and connected to the shore station through optical fibers. The two configurations are currently under construction, while taking data with the DUs already installed. ARCA is optimized for the detection of high energy neutrinos, in the range TeV$\mathrm{PeV}$. Main goals for this detector are to identify and study the high energy cosmic neutrino sources, as well as to validate the diffuse neutrino flux measured with the IceCube detector. ORCA

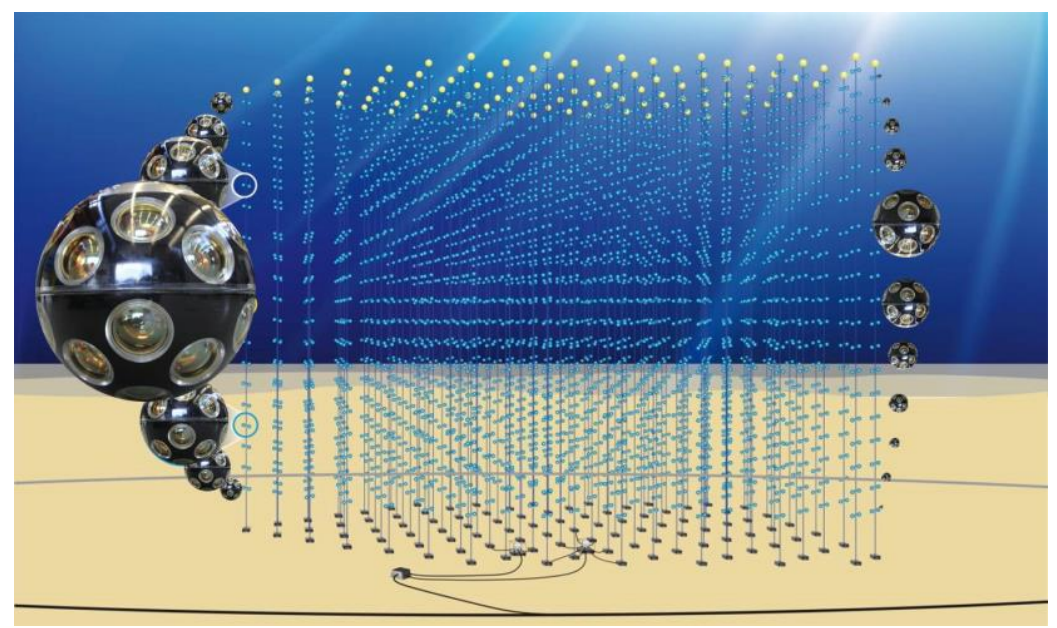

Fig. 3: The KM3NeT configuration for a block of detection, with a zoom of the DOM comprising the 31 PMTs. 
is a more compact detector that is optimized for the study of atmospheric neutrinos, having the exciting purpose to study the neutrino oscillations in order to determine the neutrino mass hierarchy. The geometries of the two configurations are very similar, the main differences being the distances between the DUs and between the DOMs, then the dimensions of the detectors.

The predominant mechanism for the energy loss of nuclearites is through elastic collisions with the atoms of the medium. As the particle passes through matter, it displaces the atoms along its path and overheats the medium in the vicinity of the particle trajectory. A part of the energy which is deposited in the interactions is dissipated as blackbody radiation in the visible spectrum. The detection of nuclearites is based on the detection of this blackbody radiation, by the PMTs of the neutrino telescope. The detection method is very similar to the neutrino detection method. When the particle passes through the instrumented volume, it generates light in the visible spectrum and this light is converted by the PMTs in electric charge, the pulse is then digitized and sent to the shore using optical fibers, where the signal will be analyzed and filtered.

\section{Monte Carlo simulations}

The Monte Carlo (MC) program used for this analysis is based on a previous code, developed for the ANTARES neutrino telescope [5], and it is adapted to simulate the KM3NeT detector and the nuclearites propagation and interaction. The MC code simulates the full geometries of the KM3NeT detector (115 lines for both ORCA and ARCA), and the characteristics of the PMTs (31 PMTs per Optical Module).

The algorithm considers an isotropic flux of downgoing nuclearites, which are uniformly distributed on a simulation hemisphere with the basis at the level of the seabed. The zenith and azimuth angles are described as follows: $\theta \in[0, \pi / 2], \varphi \in[0,2 \pi]$. The initial velocity of the nuclearites at the entry in the Earth atmosphere (approximately $50 \mathrm{~km}$ above the sea level) is considered $\beta_{0} \approx 10^{-3}$. The simulation hemisphere defines the sensitive volume of the detector. For ORCA and ARCA configurations, the radii of the sensitive volumes were taken as follows: $\mathrm{r}_{\text {hsf } \mathrm{ORCA}}=548 \mathrm{~m}, \mathrm{r}_{\text {hs__ARCA }}=912 \mathrm{~m}$, taking into account that the radii of the detectors are approximately: $\mathrm{r}_{\text {det_ORCA }}=100 \mathrm{~m}, \mathrm{r}_{\text {det_ARCA }}=500 \mathrm{~m}$. The simulation hemisphere is centered in the origin of the coordinate system, and not in the Center of Gravity (CG) of the detector. The CG of the detectors are:

- ORCA: $\mathrm{x}_{\mathrm{CG}}=0 \mathrm{~m}, \mathrm{y}_{\mathrm{CG}}=0 \mathrm{~m}, \mathrm{z}_{\mathrm{CG}}=117.1999 \mathrm{~m}$

- ARCA: $\mathrm{x}_{\mathrm{CG}}=13.8869 \mathrm{~m}, \mathrm{y}_{\mathrm{CG}}=6.7130 \mathrm{~m}, \mathrm{z}_{\mathrm{CG}}=405.9999 \mathrm{~m}$

The nuclearite events are propagated through the atmosphere and sea water until they reach the simulation hemisphere. From this point, the code generates the initial coordinates of the entry point in the sensitive volume and proceeds in time steps of $50 \mathrm{~ns}$. The propagation of the particle follows the relation (3). The algorithm searches for a luminous signal greater than 0.3 photoelectrons (pe), until the energy loss of the particle is less than $3 \mathrm{eV}$ or the particle reaches the sea bottom or exits the sensitive volume. The information obtained from the simulations that we use further are the positions of the PMTs that saw the event, the time stamp of the event and the number of 'detected' photons. The background due to ${ }^{40} \mathrm{~K}$ and to bioluminescence is not simulated. 
This preliminary analysis consists of simulated nuclearite events for ARCA and ORCA for masses in the range of $3 \cdot 10^{13}-10^{17} \mathrm{GeV}$ and an integration step of $50 \mathrm{~ns}$.

In the following, the results of a preliminary analysis of the nuclearite events simulated with the KM3NeT - ORCA and ARCA - detector configurations will be presented.

\section{Results}

This preliminary study provided us information of the expected nuclearite signal at the detector depth (number of hits, signal duration), along with the velocity of the particles at the entry in the simulation hemisphere. The residual velocities of nuclearites at the entry point in the sensitive volume were computed using the formula (3). The velocity distributions of nuclearites for ORCA and ARCA are shown in Fig. 4.
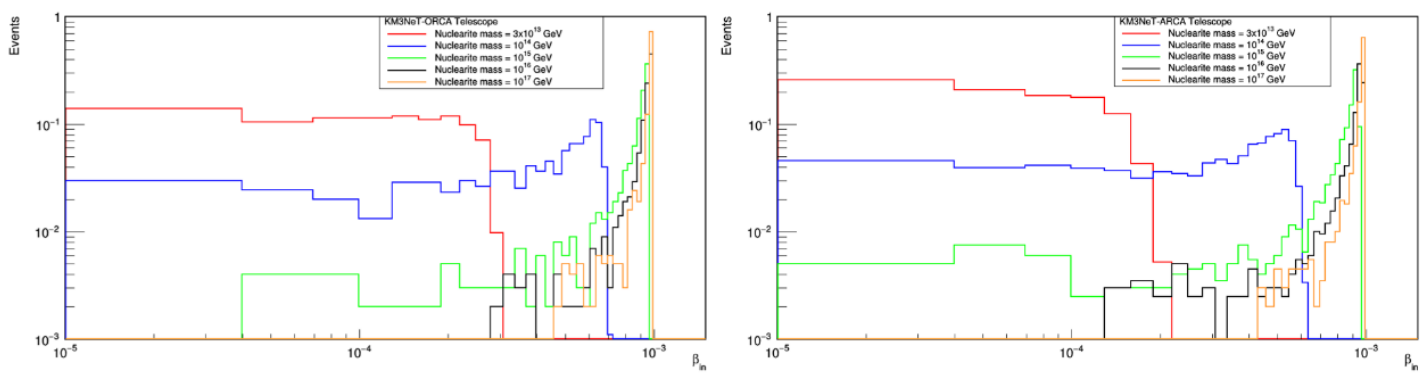

Fig. 4: Velocity distribution of nuclearites at the entry in the simulation hemisphere for KM3NeT-ORCA and KM3NeT-ARCA configurations.

In the above distributions one can observe that nuclearite events with higher masses (starting with $10^{15} \mathrm{GeV}$ ) have residual velocities at the entry in the sensitive volume approximately equal to their initial velocities at the entrance in the atmosphere.

Regarding the detector response to massive downgoing nuclearites, we obtained information about the number of hits distribution (Fig. 5).
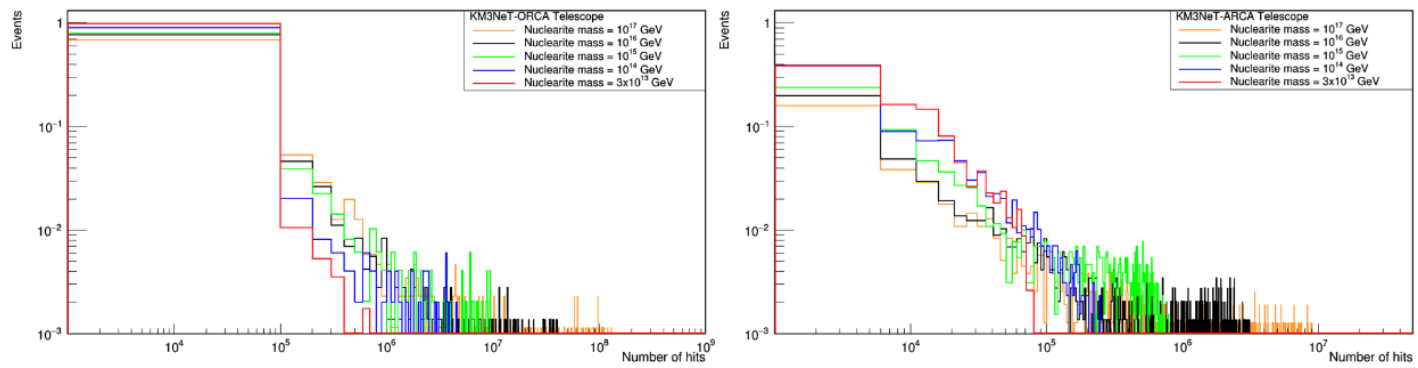

Fig. 5: The distribution of the number of hits for KM3NeT-ORCA and KM3NeT-ARCA detectors.

The above distributions made for ORCA and ARCA show that the number of hits observed depend on the photomultiplier density of the detectors. As ORCA is a denser configuration in means of PMTs, one can observe that the number of hits for the higher mass $\left(10^{17} \mathrm{GeV}\right)$ is greater in ORCA than in ARCA. For ORCA it exceeds the value of $10^{8}$, while for ARCA its value is around $10^{7}$. 
As it is clear from the above figures, the number of hits in the detector depends also on the mass of the nuclearite.

Another important information is given by the distributions of the signal duration inside the detectors (Fig. 6). The duration of the signals generated by nuclearites inside the sensitive volume is taken as the time interval between the fist and the last hit observed.
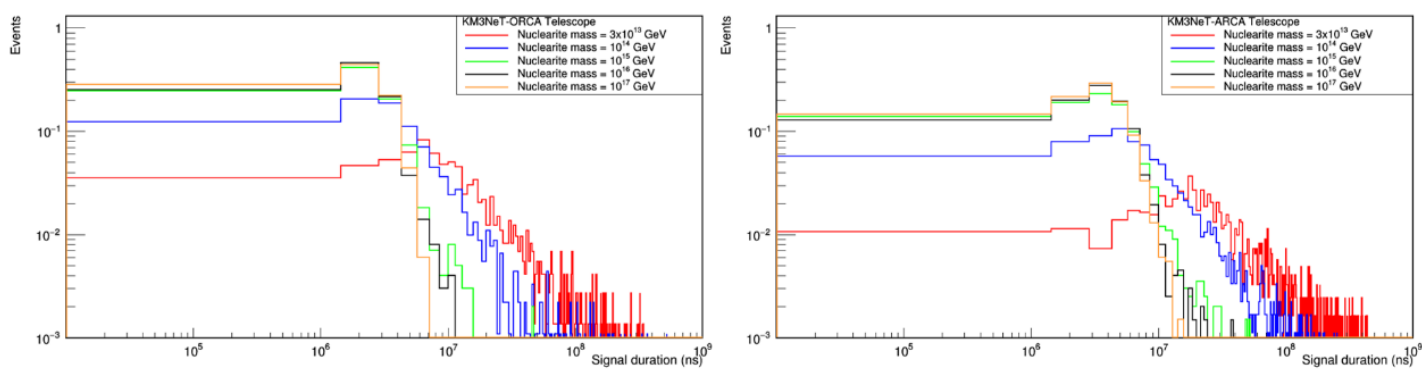

Fig. 6: The signal duration in KM3NeT-ORCA and KM3NeT-ARCA detectors.

From the distributions of the signal duration inside the detectors shown in the previous figures one can observe that there is a larger span of the signal duration distribution for the smaller masses, which are $3 \cdot 10^{13} \mathrm{GeV}$ and $10^{14} \mathrm{GeV}$. This feature can be explained by the fact that nuclearites with small masses have smaller residual velocities at the entry in the sensitive volume in comparison with the nuclearites with higher masses, as it can also be seen in Fig. 4. As they have smaller velocities, they spend more time in the sensitive volume and have a larger signal duration.

\section{Conclusions}

This is a preliminary study of the expected signal at the KM3NeT detector depth for downgoing massive nuclearites. The distributions obtained show that nuclearites propagating through underwater neutrino telescopes, such as ANTARES and KM3NeT, will induce events that will be easily recognized due to the large number of hits and a large signal duration $(>1 \mathrm{~ms})$.

\section{References}

[1] E. Witten, Cosmic Separation of Phases, Physical Review D, 30 (1984)

[2] S. A. Chin, A. K. Kerman, Possible Long-Lived Hyperstrange Multiquark Droplets, Phys. Rev. Lett. 43, 1979

[3] A. De Rujula, S. L. Glashow, Nuclearites - a novel form of cosmic radiation, Nature, 312, 1984

[4] D. Bakari, Magnetic monopoles, nuclearites, Q-balls: a qualitative picture, arXiv:hep-ex/0004019v1, 2000

[5] G. Păvălaș (ANTARES Collaboration), Search for nuclearites with the ANTARES neutrino telescope, POS(ICRC2015) 1060 


\section{Full Authors List: KM3NeT Collaboration}

M. Ageron ${ }^{1}$, S. Aiello ${ }^{2}$, A. Albert ${ }^{3,55}$, M. Alshamsi ${ }^{4}$, S. Alves Garre ${ }^{5}$, Z. Aly ${ }^{1}$, A. Ambrosone ${ }^{6,7}$, F. Ameli $^{8}$, M. Andre $^{9}$,

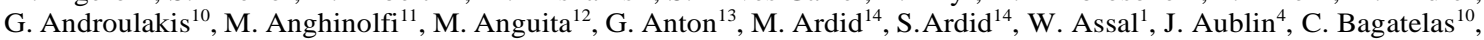
B. Baret ${ }^{4}$, S. Basegmez du Pree ${ }^{15}$, M. Bendahman ${ }^{4,16}$, F. Benfenati ${ }^{17,18}$, E. Berbee ${ }^{15}$, A. M. van den Berg ${ }^{19}$, V. Bertin ${ }^{1}$, S. Beurthey ${ }^{1}$, V. van Beveren ${ }^{15}$, S. Biagi ${ }^{20}$, M. Billault ${ }^{1}$, M. Bissinger ${ }^{13}$, M. Boettcher ${ }^{21}$, M. Bou Cabo ${ }^{22}$, J. Boumaaza ${ }^{16}$, M. Bouta ${ }^{23}$, C. Boutonnet ${ }^{4}$, G. Bouvet ${ }^{24}$, M. Bouwhuis ${ }^{15}$, C. Bozza ${ }^{25}$, H. Brânzaş $^{26}$, R. Bruijn ${ }^{15,27}$, J. Brunner ${ }^{1}$, R. Bruno ${ }^{2}$, E. Buis ${ }^{28}$, R. Buompane ${ }^{6,29}$, J. Busto ${ }^{1}$, B. Caiffi ${ }^{11}$, L. Caillat ${ }^{1}$, D. Calvo ${ }^{5}$, S. Campion ${ }^{30,8}$, A. Capone ${ }^{30,8}$, H. Carduner ${ }^{24}$, V. Carretero ${ }^{5}$, P. Castaldi ${ }^{17,31}$, S. Celli ${ }^{30,8}$, R. Cereseto ${ }^{11}$, M. Chabab ${ }^{32}$, C. Champion ${ }^{4}$, N. Chau ${ }^{4}$, A. Chen ${ }^{33}$, S. Cherubini $^{20,34}$, V. Chiarella ${ }^{35}$, T. Chiarusi ${ }^{17}$, M. Circella ${ }^{36}$, R. Cocimano ${ }^{20}$, J. A. B. Coelho ${ }^{4}$, A. Coleiro ${ }^{4}$, M. Colomer Molla ${ }^{4,5}$, S. Colonges ${ }^{4}$, R. Coniglione ${ }^{20}$, A. Cosquer ${ }^{1}$, P. Coyle ${ }^{1}$, M. Cresta $^{11}$, A. Creusot ${ }^{4}$, A. Cruz ${ }^{37}$, G. Cuttone ${ }^{20}$, A. D' Amico $^{15}$, R. Dallier ${ }^{24}$, B. De Martino ${ }^{1}$, M. De Palma ${ }^{36,38}$, I. Di Palma ${ }^{30,8}$, A. F. Díaz ${ }^{12}$, D. Diego-Tortosa ${ }^{14}$, C. Distefano ${ }^{20}$, A. Domi $^{15,27}$, C. Donzaud ${ }^{4}$, D. Dornic ${ }^{1}$, M. Dörr ${ }^{39}$, D. Drouhin ${ }^{3,55}$, T. Eberl ${ }^{13}$, A. Eddyamoui ${ }^{16}$, T. van Eeden ${ }^{15}$, D. van Eijk $^{15}$, I. El Bojaddaini ${ }^{23}$, H. Eljarrari ${ }^{16}$, D. Elsaesser ${ }^{39}$, A. Enzenhöfer ${ }^{1}$, V. Espinosa ${ }^{14}$, P. Fermani ${ }^{30,8}$, G. Ferrara ${ }^{20,34}$, M. D. Filipović $^{40}$, F. Filippini ${ }^{17,18}$, J. Fransen ${ }^{15}$, L. A. Fusco ${ }^{1}$, D. Gajanana ${ }^{15}$, T. Gal ${ }^{13}$, J. Garća Méndez ${ }^{14}$, A. Garcia Soto ${ }^{5}$, E. Garçon $^{1}$, F. Garufi ${ }^{6,7}$, C. Gatius ${ }^{15}$, N. Geißelbrecht ${ }^{13}$, L. Gialanella ${ }^{6,29}$, E. Giorgio ${ }^{20}$, S. R. Gozzini ${ }^{5}$, R. Gracia ${ }^{15}$, K. Graf ${ }^{13}$, G. Grella ${ }^{41}$, D. Guderian ${ }^{56}$, C. Guidi ${ }^{11,42}$, B. Guillon ${ }^{43}$, M. Gutiérrez ${ }^{44}$, J. Haefner ${ }^{13}$, S. Hallmann ${ }^{13}$, H. Hamdaoui ${ }^{16}$, H. van Haren $^{45}$, A. Heijboer ${ }^{15}$, A. Hekalo ${ }^{39}$, L. Hennig ${ }^{13}$, S. Henry ${ }^{1}$, J. J. Hernández-Rey ${ }^{5}$, J. Hofestädt ${ }^{13}$, F. Huang ${ }^{1}$, W. Idrissi Ibnsalih $^{6,29}$, A. Ilioni ${ }^{4}$, G. Illuminati ${ }^{17,18,4}$, C. W. James ${ }^{37}$, D. Janezashvili4' ${ }^{46}$, P. Jansweijer ${ }^{15}$, M. de Jong ${ }^{15,47}$, P. de Jong ${ }^{15,27}$, B. J. Jung ${ }^{15}$, M. Kadler ${ }^{39}$, P. Kalaczyński ${ }^{48}$, O. Kalekin ${ }^{13}$, U. F. Katz ${ }^{13}$, F. Kayzel ${ }^{15}$, P. Keller' ${ }^{1}$ N. R. Khan Chowdhury ${ }^{5}$, G. Kistauri $^{46}$, F. van der Knaap ${ }^{28}$, P. Kooijman ${ }^{27,57}$, A. Kouchner ${ }^{4,49}$, M. Kreter ${ }^{21}$, V. Kulikovskiy ${ }^{11}$, M. Labalme ${ }^{43}$, P. Lagier ${ }^{1}$, R. Lahmann ${ }^{13}$, P. Lamare ${ }^{1}$, M. Lamoureux ${ }^{14}$, G. Larosa $^{20}$, C. Lastoria ${ }^{1}$, J. Laurence ${ }^{1}$, A. Lazo ${ }^{5}$, R. Le Breton ${ }^{4}$, E. Le Guirriec $^{1}$, S. Le Stum ${ }^{1}$, G. Lehaut ${ }^{43}$, O. Leonardi ${ }^{20}$, F. Leone ${ }^{20,34}$, E. Leonora ${ }^{2}$, C. Lerouvillois ${ }^{1}, J^{2}$ Lesrel ${ }^{4}$, N. Lessing ${ }^{13}$, G. Levi ${ }^{17,18}$, M. Lincetto ${ }^{1}$, M. Lindsey Clark ${ }^{4}$, T. Lipreau ${ }^{24}$, C. LLorens Alvarez ${ }^{14}$, A. Lonardo ${ }^{8}$, F. Longhitano ${ }^{2}$, D. LopezCoto $^{44}$, N. Lumb ${ }^{1}$, L. Maderer ${ }^{4}$, J. Majumdar ${ }^{15}$, J. Mańczak ${ }^{5}$, A. Margiotta ${ }^{17,18}$, A. Marinelli6, A. Marini ${ }^{1}$, C. Markou ${ }^{10}$, L. $^{6}$ Martin $^{24}$, J. A. Martínez-Mora ${ }^{14}$, A. Martini ${ }^{35}$, F. Marzaioli ${ }^{6,29}$, S. Mastroianni' ${ }^{6}$, K. W. Melis ${ }^{15}$, G. Miele ${ }^{6,7}$, P. Migliozzi ${ }^{6}$, E. Migneco $^{20}$, P. Mijakowski ${ }^{48}$, L. S. Miranda ${ }^{50}$, C. M. Mollo, ${ }^{6}$ M. Mongelli ${ }^{36}$, A. Moussa ${ }^{23}$, R. Muller ${ }^{15}$, P. Musico ${ }^{11}$, M. Musumeci $^{20}$, L. Nauta ${ }^{15}$, S. $\mathrm{Navas}^{44}$, C. A. Nicolau ${ }^{8}$, B. Nkosi ${ }^{33}$, B.ÓFearraigh ${ }^{15,27}$, M. O’Sullivan ${ }^{37}$, A. Orlando ${ }^{20}$, G. Ottonello $^{11}$, S. Ottonello ${ }^{11}$, J. Palacios González ${ }^{5}$, G. Papalashvili ${ }^{46}$, R. Papaleo ${ }^{20}$, C. Pastore ${ }^{36}$, A. M. Păun ${ }^{26}$, G. E. Păvălaş ${ }^{26}$, G. Pellegrini i ${ }^{17}$, C. Pellegrino ${ }^{18,58}$, M. Perrin-Terrin ${ }^{1}$, V. Pestel ${ }^{15}$, P. Piattelli ${ }^{20}$, C. Pieterse ${ }^{5}$, O. Pisanti ${ }^{6,7}$, C. Poirè $^{14}$, V. Popa $^{26}$, T. Pradier ${ }^{3}$, F. Pratolongo ${ }^{11}$, I. Probst ${ }^{13}$, G. Pühlhofer ${ }^{51}$, S. Pulvirenti ${ }^{20}$, G. Quéméner ${ }^{43}$, N. Randazzo ${ }^{2}$, A. $^{\prime}$ Rapicavoli $^{34}$, S. Razzaque ${ }^{50}$, D. Real ${ }^{5}$, S. Reck ${ }^{13}$, G. Riccobene ${ }^{20}$, L. Rigalleau ${ }^{24}$, A. Romanov ${ }^{11,42}$, A. Rovellii ${ }^{20}$, J. Royon ${ }^{1}$, F. Salesa Greus ${ }^{5}$, D. F. E. Samtleben ${ }^{15,47}$, A. Sánchez Losa ${ }^{36,5}$, M. Sanguineti ${ }^{11,42}$, A. Santangelo ${ }^{51}$, D. Santonocito ${ }^{20}$, P. Sapienza $^{20}$, J. Schmelling ${ }^{15}$, J. Schnabel ${ }^{13}$, M. F. Schneider ${ }^{13}$, J. Schumann ${ }^{13}$, H. M. Schutte ${ }^{21}$, J. Seneca ${ }^{15}$, I. Sgura ${ }^{36}$, R. Shanidze ${ }^{46}$, A. Sharma ${ }^{52}$, A. Sinopoulou ${ }^{10}$, B. Spisso ${ }^{41,6}$, M. Spurio ${ }^{17,18}$, D. Stavropoulos ${ }^{10}$, J. Steijger ${ }^{15}$, S. M. Stellacci ${ }^{41,6}$, M. Taiuti ${ }^{11,42}$, F. Tatone ${ }^{36}$, Y. Tayalati ${ }^{16}$, E. Tenllado ${ }^{44}$, D. Tézier ${ }^{1}$, T. Thakore ${ }^{5}$, S. Theraube ${ }^{1}$, H. Thiersen ${ }^{21}$, P. Timmer ${ }^{15}$, S. Tingay ${ }^{37}$, S. Tsagkli ${ }^{10}$, V. Tsourapis ${ }^{10}$, E. Tzamariudaki ${ }^{10}$, D. Tzanetatos ${ }^{10}$, C. Valieri ${ }^{17}$, V. Van Elewyck ${ }^{4,49}$, G. Vasileiadis ${ }^{53}$, F. Ver- $^{2}$ sari $^{17,18}$, S. Viola ${ }^{20}$, D. Vivolo ${ }^{6,29}$, G. de Wasseige ${ }^{4}$, J. Wilms ${ }^{54}$, R. Wojaczyński ${ }^{48}$,E. de Wolf ${ }^{15,27}$, T. Yousfi ${ }^{23}$, S. Zavatarelli ${ }^{11}$, A. Zegarellii ${ }^{30,8}$,D. Zito ${ }^{20}$, J. D. Zornoza ${ }^{5}$, J. Zúñiga ${ }^{5}$, N. Zywucka ${ }^{21}$.

${ }^{1}$ Aix Marseille Univ, CNRS/IN2P3, CPPM, Marseille, France.

${ }^{2}$ INFN, Sezione di Catania, Via Santa Sofia 64, Catania, 95123 Italy.

${ }^{3}$ Université de Strasbourg, CNRS, IPHC UMR 7178, F-67000 Strasbourg, France.

${ }^{4}$ Université de Paris, CNRS, Astroparticule et Cosmologie, F-75013 Paris, France.

${ }^{5}$ IFIC - Instituto de Física Corpuscular (CSIC - Universitat de València), c/Catedrático José Beltrán, 2, 46980 Paterna, Valencia, Spain.

${ }^{6}$ INFN, Sezione di Napoli, Complesso Universitario di Monte S. Angelo, Via Cintia ed. G, Napoli, 80126 Italy.

${ }^{7}$ Università di Napoli "Federico II", Dip. Scienze Fisiche "E. Pancini”, Complesso Universitario di Monte S. Angelo, Via Cintia ed. G, Napoli, 80126 Italy.

${ }^{8}$ INFN, Sezione di Roma, Piazzale Aldo Moro 2, Roma, 00185 Italy.

${ }^{9}$ Universitat Politècnica de Catalunya, Laboratori d'Aplicacions Bioacústiques, Centre Tecnològic de Vi- lanova i la Geltrú, Avda. Rambla Exposició, s/n, Vilanova i la Geltrú, 08800 Spain.

${ }^{10}$ NCSR Demokritos, Institute of Nuclear and Particle Physics, Ag. Paraskevi Attikis, Athens, 15310 Greece.

${ }^{11}$ INFN, Sezione di Genova, Via Dodecaneso 33, Genova, 16146 Italy.

${ }^{12}$ University of Granada, Dept. of Computer Architecture and Technology/CITIC, 18071 Granada, Spain.

${ }^{13}$ Friedrich-Alexander-Universität Erlangen-Nürnberg, Erlangen Centre for Astroparticle Physics, Erwin- Rommel-Straße 1, 91058 Erlangen, Germany.

${ }^{14}$ Universitat Politècnica de València, Instituto de Investigación para la Gestión Integrada de las Zonas Costeras, C/ Paranimf, 1, Gandia, 46730 Spain.

${ }^{15}$ Nikhef, National Institute for Subatomic Physics, PO Box 41882, Amsterdam, 1009 DB Netherlands.

${ }^{16}$ University Mohammed V in Rabat, Faculty of Sciences, 4 av. Ibn Battouta, B.P. 1014, R.P. 10000Rabat, Morocco.

${ }^{17}$ INFN, Sezione di Bologna, v.le C. Berti-Pichat, 6/2, Bologna, 40127 Italy.

${ }^{18}$ Università di Bologna, Dipartimento di Fisicae Astronomia, v.le C. Berti-Pichat, 6/2, Bologna, 40127 Italy.

${ }^{19}$ KVI-CART University of Groningen, Groningen, the Netherlands.

${ }^{20}$ INFN, Laboratori Nazionali del Sud, Via S. Sofia 62, Catania, 95123 Italy.

${ }^{21}$ North-West University, Centre for Space Research, Private Bag X6001, Potchefstroom, 2520 SouthAfrica.

${ }^{22}$ Instituto Español de Oceanografía, Unidad Mixta IEO-UPV, C/ Paranimf, 1, Gandia, 46730 Spain.

${ }^{1}$ also at Dipartimento di Fisica, INFN Sezione di Padova and Università di Padova, I-35131, Padova, Italy 
${ }^{23}$ University Mohammed I, Faculty of Sciences, BV Mohammed VI, B.P. 717, R.P. 60000 Oujda, Morocco.

${ }^{24}$ Subatech, IMT Atlantique, IN2P3-CNRS, Université de Nantes, 4 rue Alfred Kastler - La Chantrerie, Nantes, BP 2072244307 France.

${ }^{25}$ Università di Salerno e INFN Gruppo Collegato di Salerno, Dipartimento di Matematica, Via Giovanni Paolo II 132, Fisciano, 84084 Italy.

${ }^{26}$ ISS, Atomistilor 409, Măgurele, RO-077125 Romania.

${ }^{27}$ University of Amsterdam, Institute of Physics/IHEF, PO Box 94216, Amsterdam, 1090 GE Nether-lands.

${ }^{28}$ TNO, Technical Sciences, PO Box 155, Delft, 2600 AD Netherlands.

${ }^{29}$ Università degli Studi della Campania "Luigi Vanvitelli”, Dipartimento di Matematica e Fisica, viale Lincoln 5, Caserta, 81100 Italy.

${ }^{30}$ Università La Sapienza, Dipartimento di Fisica, Piazzale Aldo Moro 2, Roma, 00185 Italy.

${ }^{31}$ Università di Bologna, Dipartimento di Ingegneria dell'Energia Elettrica e dell'Informazione "Guglielmo Marconi”, Via dell'Università 50, Cesena, 47521 Italia.

${ }^{32}$ Cadi Ayyad University, Physics Department, Faculty of Science Semlalia, Av. My Abdellah, P.O.B.2390, Marrakech, 40000 Morocco.

${ }^{33}$ University of the Witwatersrand, School of Physics, Private Bag 3, Johannesburg, Wits 2050 SouthAfrica.

${ }^{34}$ Università di Catania, Dipartimento di Fisica e Astronomia "Ettore Majorana”, Via Santa Sofia 64, Catania, 95123 Italy.

${ }^{35}$ INFN, LNF, Via Enrico Fermi, 40, Frascati, 00044 Italy.

${ }^{36}$ INFN, Sezione di Bari, via Orabona, 4, Bari, 70125 Italy.

${ }^{37}$ International Centre for Radio Astronomy Research, Curtin University, Bentley, WA 6102, Australia.

${ }^{38}$ University of Bari, Via Amendola 173, Bari, 70126 Italy.

${ }^{39}$ University Würzburg, Emil-Fischer-Straße 31, Würzburg, 97074 Germany.

${ }^{40}$ Western Sydney University, School of Computing, Engineering and Mathematics, Locked Bag 1797,Penrith, NSW 2751 Australia.

${ }^{41}$ Università di Salerno e INFN Gruppo Collegato di Salerno, Dipartimento di Fisica, Via Giovanni Paolo II 132, Fisciano, 84084 Italy.

${ }^{42}$ Università di Genova, Via Dodecaneso 33, Genova, 16146 Italy.

${ }^{43}$ Normandie Univ, ENSICAEN, UNICAEN, CNRS/IN2P3, LPC Caen, LPCCAEN, 6 boulevard Maréchal Juin, Caen, 14050 France.

${ }^{44}$ University of Granada, Dpto. de Física Teórica y del Cosmos \& C.A.F.P.E., 18071 Granada, Spain.

${ }^{45}$ NIOZ (Royal Netherlands Institute for Sea Research), PO Box 59, Den Burg, Texel, 1790 AB, theNetherlands.

${ }^{46}$ Tbilisi State University, Department of Physics, 3, Chavchavadze Ave., Tbilisi, 0179 Georgia

${ }^{47}$ Leiden University, Leiden Institute of Physics, PO Box 9504, Leiden, 2300 RA Netherlands.

${ }^{48}$ National Centre for Nuclear Research, 02-093 Warsaw, Poland.

${ }^{49}$ Institut Universitaire de France, 1 rue Descartes, Paris, 75005 France.

${ }^{50}$ University of Johannesburg, Department Physics, PO Box 524, Auckland Park, 2006 South Africa.

${ }^{51}$ Eberhard Karls Universität Tübingen, Institut für Astronomie und Astrophysik, Sand 1, Tübingen, 72076 Germany.

${ }^{52}$ Università di Pisa, Dipartimento di Fisica, Largo Bruno Pontecorvo 3, Pisa, 56127 Italy.

${ }^{53}$ Laboratoire Univers et Particules de Montpellier, Place Eugène Bataillon - CC 72, Montpellier Cédex 05, 34095 France.

${ }^{54}$ Friedrich-Alexander-Universität Erlangen-Nürnberg, Remeis Sternwarte, Sternwartstraße 7, 96049 Bam- berg, Germany.

${ }^{55}$ Université de Haute Alsace, 68100 Mulhouse Cedex, France.

${ }^{56}$ University of Münster, Institut für Kernphysik, Wilhelm-Klemm-Str. 9, Münster, 48149 Germany.

${ }^{57}$ Utrecht University, Department of Physics and Astronomy, PO Box 80000, Utrecht, 3508 TA Nether-lands.

${ }^{58}$ INFN, CNAF, v.le C. Berti-Pichat, 6/2, Bologna, 40127 Italy. 